\title{
2011 American Academy of Optometry (AAO) in Boston
}

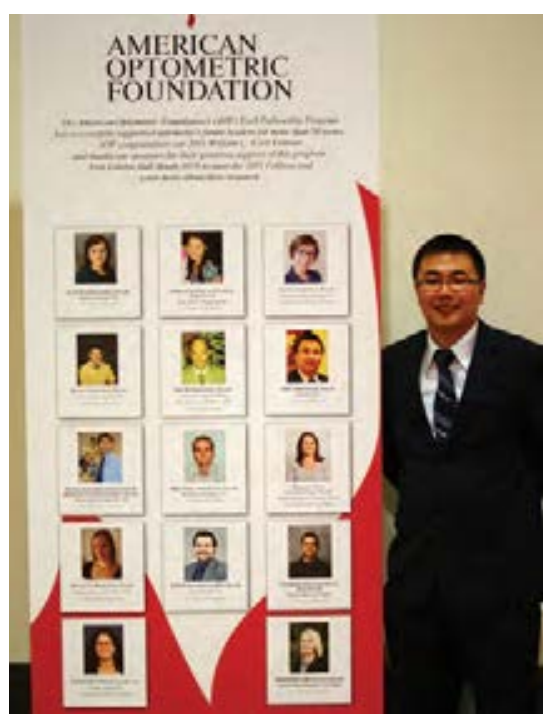

Alex Hui (UW) received the Ezell Travel Fellowship from the American Optometric Foundation (AOF) at the AAO meeting

BY ETTY BITTON, OD, MSC, FAAO \& DEBORAH JONES FCOptom, DipCLP, FAAO

nother year, another meeting! Why Ado they seem to come around more quickly from year to year? The two Canadian schools once again were well represented at this key optometry meeting, held last October in Boston. The meeting attracted a total of 5693 attendees, the second largest of all time, and a record-breaking, 1094 students. Optometrists, vision scientists, professors, residents and students congregated for the 4-day meeting packed full of information. Conferences, scientific presentations (paper and poster presentations), workshops, special symposiums, and the latest technologies and products in the exhibit hall were available for the meeting participants.

Faculty, graduate and optometry students from both Canadian schools also participated in the meeting with contributions to lectures and scientific presentations, as shown in Table 1 and 2.
Table 1: École d’optométrie, Université de Montréal participation at the 2011 AAO Meeting

\begin{tabular}{|c|c|}
\hline \multicolumn{2}{|r|}{ FACULTY } \\
\hline AUTHOR & TITLE \\
\hline $\begin{array}{l}\text { Bitton } \mathrm{E} \\
\text { Bitton } \mathrm{E}, \text { Landreville MP, } \\
\text { Forget } \mathrm{MC}\end{array}$ & $\begin{array}{l}\text { A CLOSER LOOK AT THE OCULAR TEAR FILM (conference } 1 \text { hr) } \\
\text { INFLUENCE OF EYE POSITION ON THE SCHIRMER TEAR TEST (paper) }\end{array}$ \\
\hline $\begin{array}{l}\text { Brulé J, Tousignant B } \\
\text { Brulé J, Duguay C, } \\
\text { Larouche J } \\
\text { Brulé J, Larouche J, } \\
\text { Duguay C }\end{array}$ & $\begin{array}{l}\text { EVIDENCE-BASED TOOL TO TRAIN EDUCATORS IN MULTIPLE-CHOICE QUESTION } \\
\text { WRITING IN RESOURCE-LIMITED SETTINGS: A PILOT STUDY (poster) } \\
\text { AWARENESS OF MODALITIES OF EYE EXAM IN QUEBEC, CANADA (poster) } \\
\text { IDENTIFYING THE NEEDS FOR OCULAR HEALTH EDUCATION IN QUEBEC, CANADA } \\
\text { (poster) }\end{array}$ \\
\hline Faubert $J$ & PERCEPTUAL-COGNITIVE SPEED TRAINING WITH PROFESSIONAL ATHLETES (poster) \\
\hline $\begin{array}{l}\text { Frenette B, } \\
\text { Michaud L }\end{array}$ & $\begin{array}{l}\text { IMPROVEMENT OF CONTACT LENS COMFORT WITH THE USE OF A SODIUM } \\
\text { HYALURONATE (SH) DROP ON THE LENS BEFORE INSERTION (poster) }\end{array}$ \\
\hline $\begin{array}{l}\text { Kergoat H, LovasikJV, } \\
\text { Kergoat MJ, Racine N, } \\
\text { Parent M }\end{array}$ & RETINAL METABOLIC REGULATION WITH AGE (poster) \\
\hline $\begin{array}{l}\text { Michaud L } \\
\text { Michaud L, Bitton E, et al. } \\
\text { Michaud L }\end{array}$ & $\begin{array}{l}\text { MODERN RGP LENS DESIGNS FOR DAY-TO-DAY PRACTICE: A CLINICAL GRAND } \\
\text { ROUND (conference } 1 \mathrm{hr} \text { ) } \\
\text { GRAND ROUNDS I: First Report of Ocular Dryness as Related to Maple Syrup Urine } \\
\text { Disease (MSUD) (conference } 2 \mathrm{hr} \text { ) } \\
\text { TOPS: A NEW CLINICAL FINDING RELATED TO FABRY'S DISEASE (poster) }\end{array}$ \\
\hline Overbury O & $\begin{array}{l}\text { A NEW PhD PROGRAM IN VISION SCIENCE AT THE UNIVERSITY OF MONTREAL } \\
\text { (poster) }\end{array}$ \\
\hline $\begin{array}{l}\text { Renaud J, Overbury O, } \\
\text { Durand MJ }\end{array}$ & $\begin{array}{l}\text { WHAT EXPLAINS GOOD SUBJECTIVE QUALITY OF LIFE OF OLDER ADULTS WITH } \\
\text { VISUAL IMPAIRMENT? (poster) }\end{array}$ \\
\hline
\end{tabular}

\section{OPTOMETRY STUDENTS}

\begin{tabular}{|l|l|}
\hline AUTHOR & TITLE \\
\hline $\begin{array}{l}\text { Bachir V, Rubino L, } \\
\text { Warde R, Lovasik JV, } \\
\text { Kergoat H }\end{array}$ & $\begin{array}{l}\text { THE EFFECTS OF PANRETINAL PHOTOCOAGULATION ON NEUROVASCULAR } \\
\text { COUPLING IN THE HUMAN DIABETIC EYE (poster) }\end{array}$ \\
\hline $\begin{array}{l}\text { Bédard E, Michaud L, } \\
\text { Brazeau D, Pop M }\end{array}$ & COMPARATIVE STUDY OF 3 MODES OF TREATMENT FOR KERATOCONUS (poster) \\
\hline $\begin{array}{l}\text { Deschambault E, } \\
\text { Abboud C, Brûlé J }\end{array}$ & $\begin{array}{l}\text { SMOKING CESSATION COUNSELLING: PRACTICES OF QUÉBEC OPTOMETRISTS } \\
\text { (poster) }\end{array}$ \\
\hline $\begin{array}{l}\text { Hong Y, Bitton E } \\
\text { ACQUIRED FACIAL NERVE PALSY FOLLOWED BY HERPES ZOSTER INFECTION: } \\
\text { MANAGEMENT OF OCULAR SYMPTOMOLOGY (pOSTEr) }\end{array}$ \\
\hline $\begin{array}{l}\text { Samaha D, Lafleur G, } \\
\text { Michaud L }\end{array}$ & $\begin{array}{l}\text { HYDROGEN PEROXIDE AS A BETTER ALTERNATIVE CARE REGIMEN FOR RGP AND } \\
\text { RIGID CONTACT LENSES (paper) }\end{array}$ \\
\hline Xie T, Bitton E & $\begin{array}{l}\text { AN ATYPICAL CASE OF HLA-B27- ASSOCIATED UVEITIS WITH HYPOPYON AND } \\
\text { POSTERIOR SEGMENT INVOLVEMENT (pOSTEr) }\end{array}$ \\
\hline
\end{tabular}

Continued 
Several awards were handed out at the AAO meeting including the Carl Zeiss Vision Fellowship to optometry students Claudine Courey (UM) and Mariam Nahal (UW). William C. Ezell Fellowships for graduate work was awarded to Jean-Marie Hanssens (UM) and Alex Hui (UW). Several travel fellowships were also available to help optometry and graduate students attend the meeting. These included the Essilor Student Travel Fellowship awarded to Samar Farhat (UM) and Tyler Anderson (UW); The Vision Care Institute ${ }^{\mathrm{TM}}$, LLC Travel Fellowship was awarded to Vanessa Bachir, Estefania Chriqui, Reza Abbas Farishta (UM) and Alan Ng, Salsabeel Jadi and Subam Basuthka (UW). The Brazelton Low Vision Student Travel Fellowship was awarded to Lea Gagnon (UM), the Irvin Borish Student Travel Fellowship was awarded to Judith Renaud (UM) and the Section on Cornea, Contact Lenses \& Refractive Technologies Resident Travel Fellowship was awarded to Richard Warde (UM). Dr. Thom Freddo (UW professor) was appointed to the Board of Regents of Beta Sigma Kappa.

The annual meeting is also the time where new Fellows of the AAO (FAAO) get inducted. This process is a peer-recognition whereby an optometrist or vision scientist demonstrates their knowledge, contributions and leadership to a committee. The annual meeting culminates this effort with a final interview process and those that successfully complete the process are awarded a FAAO at the meeting. This year 194 new Fellows were inducted with six Canadians including Dr. Walter Wittich (UM and presently at the MAB-MacKay Rehabilitation Center), Dr. Doerte Luensmann (UW-CCLR), Dr Marc Schultze (UWCCLR), Dr Sara Maclver (UW), Dr Mark Eltis (Toronto, Ontario) and Dr David Schwirtz (Surrey, British Columbia). Congratulations to all the new Fellows!

Next year the meeting will be held in Phoenix, Arizona on October 24-27, 2012. Looking forward to seeing you there !

Table 1: École d'optométrie, Université de Montréal (continued from p.11)

\begin{tabular}{|c|c|}
\hline \multicolumn{2}{|r|}{ GRADUATE STUDENTS } \\
\hline AUTHOR & TITLE \\
\hline \multirow[t]{2}{*}{ Abbas R } & $\begin{array}{l}\text { EFFECTS ON THE VISUAL CORTEX OF ELECTRICAL AND VISUAL STIMULATION OF } \\
\text { THALAMIC NUCLEI REVEALED BY VSD IMAGING (poster) }\end{array}$ \\
\hline & $\begin{array}{l}\text { RESPONSES OF THE STRIATE AND EXTRA STRIATE CORTEX TO PULVINAR AND LGN } \\
\text { STIMULATION IN THREE SHREWS (poster) }\end{array}$ \\
\hline $\begin{array}{l}\text { Chriqui E, Kergoat MJ, } \\
\text { Champoux N, } \\
\text { Leclerc BS, Kergoat H }\end{array}$ & $\begin{array}{l}\text { ASSESSMENT OF VISUAL ACUITY OF COGNITIVELY IMPAIRED OLDER INDIVIDUALS } \\
\text { RESIDING IN LONG-TERM CARE FACILITY (poster) }\end{array}$ \\
\hline $\begin{array}{l}\text { Gagnon L, Aumond S, } \\
\text { Huppe A, Ptito, M }\end{array}$ & HOW TO IMPROVE TACTILE MAPS: LESSONS FROM BLINDNESS! (poster) \\
\hline $\begin{array}{l}\text { Giraudet G, Miconi C, } \\
\text { Hanssens JM, Faubert J }\end{array}$ & $\begin{array}{l}\text { ROLE OF BINOCULAR DISPARITIES IN THE VISUAL CONTROL OF QUIET STANCE } \\
\text { (poster) }\end{array}$ \\
\hline $\begin{array}{l}\text { Hanssens JM, Giraudet G, } \\
\text { Allard R, Faubert J }\end{array}$ & $\begin{array}{l}\text { MODULATING SOMATO-SENSORY INPUT INCREASES VISUAL DEPENDENCY FOR } \\
\text { POSTURAL CONTROL (poster) }\end{array}$ \\
\hline
\end{tabular}

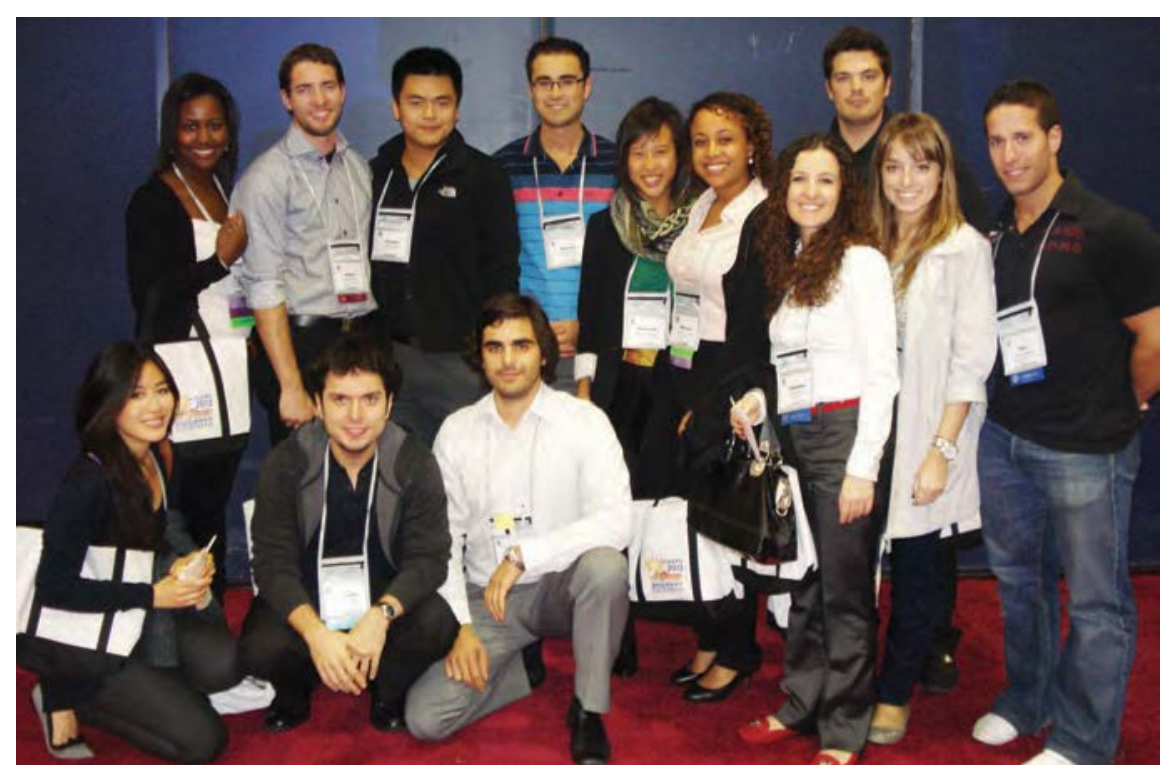

Student attendees from Montreal at the AAO meeting

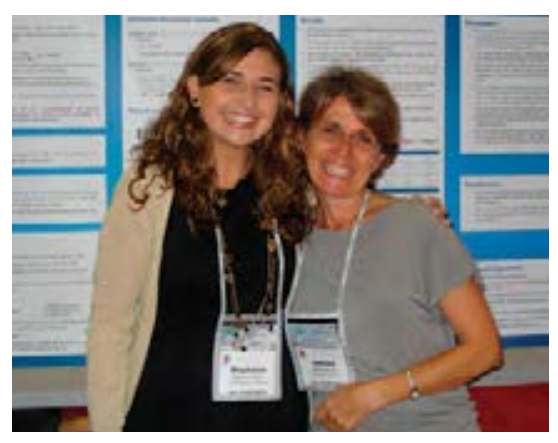

Master's student Dr. Estefania Chriqui and supervisor Dr. Hélène Kergoat (UM) presenting at the scientific section of the meeting.

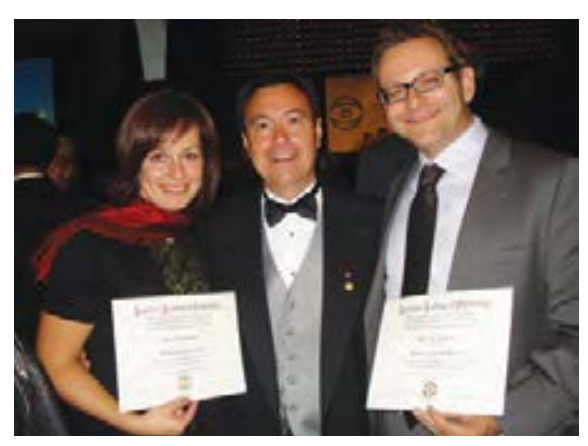

Dr. Lyndon Jones flanked by new AAO Fellows, Dr. Doerte Luensmann and Dr. Marc Schultze (UW) 
Table 2: School of Optometry, University of Waterloo participation at the 2011 AAO Meeting

\begin{tabular}{|c|c|}
\hline AUTHOR & TITLE \\
\hline $\begin{array}{l}\text { Alex Hui* (first author) } \\
\text { Heather Sheardown, } \\
\text { Lyndon Jones }\end{array}$ & $\begin{array}{l}\text { MOLECULAR IMPRINTED SILICONE } \\
\text { HYDROGEL MATERIALS FOR } \\
\text { CIPROFLOXACIN DRUG DELIVERY (paper) }\end{array}$ \\
\hline $\begin{array}{l}\text { Kathy Dumbleton (first author) } \\
\text { Craig Woods, Lyndon Jones, } \\
\text { Desmond Fonn }\end{array}$ & $\begin{array}{l}\text { A SURVEY TO INVESTIGATE LAPSED } \\
\text { CONTACT LENS WEARERS IN CANADA } \\
\text { (paper) }\end{array}$ \\
\hline $\begin{array}{l}\text { Kathy Dumbleton (first author) } \\
\text { Craig Woods, Lyndon Jones, } \\
\text { Desmond Fonn }\end{array}$ & $\begin{array}{l}\text { COMPARING CONTACT LENS COMPLIANCE } \\
\text { AND COMPLICATIONS IN A UNIVERSITY } \\
\text { CLINIC WITH PRIVATE OPTOMETRY OFFICES } \\
\text { (paper) }\end{array}$ \\
\hline $\begin{array}{l}\text { Mike Woods (first author) } \\
\text { Kathy Dumbleton, Craig Woods, } \\
\text { Lyndon Jones, Desmond Fonn }\end{array}$ & $\begin{array}{l}\text { DO CONTACT LENS WEARERS REMEMBER } \\
\text { WHAT PRODUCTS THEY ARE USING? (poster) }\end{array}$ \\
\hline $\begin{array}{l}\text { Doerte Luensmann (first author) } \\
\text { Nancy Keir, Megan Despres, } \\
\text { Doris Richter, Craig Woods, } \\
\text { Desmond Fonn }\end{array}$ & $\begin{array}{l}\text { IN VIVO WETTABILITY CHANGES OVER } 3 \\
\text { DAYS USING DAILY DISPOSABLE CONTACT } \\
\text { LENSES (poster) }\end{array}$ \\
\hline $\begin{array}{l}\text { Alan Ng (first author) } \\
\text { Miriam Heynen, Lyndon Jones }\end{array}$ & $\begin{array}{l}\text { THE IMPACT OF LACTOFERRIN AND LIPIDS } \\
\text { ON KINETIC LYSOZYME DEPOSITION ON } \\
\text { CONTACT LENSES (paper) }\end{array}$ \\
\hline $\begin{array}{l}\text { Sarah Guthrie (first author) } \\
\text { Jill Woods, Nancy Keir, Vivian } \\
\text { Choh, Sally Dellehay, Mark Tyson, } \\
\text { Richard Griffin, Lyndon Jones, } \\
\text { Elizabeth Irving }\end{array}$ & $\begin{array}{l}\text { CONTROLLING LENS INDUCED MYOPIA IN } \\
\text { CHICKENS WITH PERIPHERAL LENS DESIGN } \\
\text { (paper) }\end{array}$ \\
\hline $\begin{array}{l}\text { Subam Basuthkar (first author) } \\
\text { Trefford Simpson }\end{array}$ & $\begin{array}{l}\text { DOES DEFOCUS CONTRIBUTE TO OCULAR } \\
\text { DISCOMFORT? (paper) }\end{array}$ \\
\hline $\begin{array}{l}\text { Marc Schulze (first author) } \\
\text { Trefford Simpson, Ping Situ, } \\
\text { Kara Menzies, Hendrik Walther, } \\
\text { Lyndon Jones }\end{array}$ & $\begin{array}{l}\text { EFFECTS OF MAGNIFICATION ON TEAR } \\
\text { MENISCUS PARAMETERS USING OPTICAL } \\
\text { COHERENCE TOMOGRAPHY (OCT) IMAGES } \\
\text { (poster) }\end{array}$ \\
\hline $\begin{array}{l}\text { Patricia Hrynchak (first author) } \\
\text { Carolyn Machan, Elizabeth Irving }\end{array}$ & $\begin{array}{l}\text { NEAR PHORIA AND STRABISMUS AS A } \\
\text { FUNCTION OF AGE IN A CLINIC } \\
\text { POPULATION (poster) }\end{array}$ \\
\hline $\begin{array}{l}\text { Norris Lam (first author) } \\
\text { Alison Leong, Susan Leat }\end{array}$ & $\begin{array}{l}\text { LOW VISION SERVICE PROVISION BY } \\
\text { OPTOMETRISTS - A CANADIAN SURVEY } \\
\text { (Poster) }\end{array}$ \\
\hline $\begin{array}{l}\text { Balsam Alabdulkader } \\
\text { (first author) } \\
\text { Susan Leat }\end{array}$ & $\begin{array}{l}\text { THE EFFECTIVENESS OF READING ADDI- } \\
\text { TIONS FOR CHILDREN AND YOUNG ADULTS } \\
\text { WITH LOW VISION (paper) }\end{array}$ \\
\hline Susan Leat & $\begin{array}{l}\text { PEDIATRIC LOWVISION MANAGEMENT - } \\
\text { MAKING A START - CE lecture chair and } \\
\text { co-founder of this group (inaugural meeting } \\
\text { this year) }\end{array}$ \\
\hline Susan Leat & $\begin{array}{l}\text { VISION IN AGING SIG - CE lecture chair and } \\
\text { co-founder of this group (inaugural meeting } \\
\text { this year) }\end{array}$ \\
\hline $\begin{array}{l}\text { Salsabeel Jadi (first author) } \\
\text { Miriam Heynen, } \\
\text { Doerte Luensmann, } \\
\text { Lyndon Jones }\end{array}$ & $\begin{array}{l}\text { INCUBATION SOLUTION COMPOSITION } \\
\text { IMPACTS IN VITRO PROTEIN UPTAKE TO } \\
\text { SILICONE HYDROGEL CONTACT LENSES } \\
\text { (paper) }\end{array}$ \\
\hline $\begin{array}{l}\text { Carolyn Machan (first author) } \\
\text { Patricia Hrynchak, } \\
\text { Elizabeth Irving }\end{array}$ & $\begin{array}{l}\text { STATIN USE, TYPE } 2 \text { DIABETES AND AGE- } \\
\text { RELATED CATARACT:WATERLOO EYE STUDY } \\
\text { (paper) }\end{array}$ \\
\hline Thomas Freddo & $\begin{array}{l}\text { THE MEDICAL WORK-UP OF THE RED EYE - } \\
\text { CE(TQ) Lecture }\end{array}$ \\
\hline Thomas Freddo & $\begin{array}{l}\text { CONJUNCTIVAL LESIONS: LINKS TO } \\
\text { SYSTEMIC DISEASE - CE(TQ) Lecture }\end{array}$ \\
\hline
\end{tabular}

\begin{tabular}{|c|c|}
\hline AUTHOR & TITLE \\
\hline $\begin{array}{l}\text { Elizabeth Irving (first author) } \\
\text { Carolyn Machan, Andrea Mittles- } \\
\text { taedt, Patricia Hrynchak }\end{array}$ & $\begin{array}{l}\text { DIFFERENCE BETWEEN PRESENTING AND } \\
\text { BEST CORRECTED VISUAL ACUITY AS A } \\
\text { FUNCTION OF AGE (poster) }\end{array}$ \\
\hline $\begin{array}{l}\text { Elizabeth Irving (first author) } \\
\text { Carolyn Machan, Patricia } \\
\text { Hrynchak }\end{array}$ & $\begin{array}{l}\text { ASSOCIATIONS BETWEEN REFRACTIVE } \\
\text { ERROR, NEAR PHORIA AND AGE (poster) }\end{array}$ \\
\hline $\begin{array}{l}\text { B. Ralph Chou (first author) } \\
\text { Jeffery K. Hovis }\end{array}$ & $\begin{array}{l}\text { OCULAR HAZARD OF A THERMAL LANCE } \\
\text { (poster) }\end{array}$ \\
\hline $\begin{array}{l}\text { Craig Woods (first author) } \\
\text { Nancy Keir, Doerte Luensmann, } \\
\text { Desmond Fonn }\end{array}$ & $\begin{array}{l}\text { VISUAL PERFORMANCE OF MULTIFOCAL } \\
\text { CONTACT LENSES (PAPER) }\end{array}$ \\
\hline $\begin{array}{l}\text { Sruthi Srinivasan (first author) } \\
\text { Kara Menzies, Luigina Sorbara, } \\
\text { Lyndon W Jones }\end{array}$ & $\begin{array}{l}\text { IMAGING MEIBOMIAN GLAND STRUCTURES } \\
\text { USING THE OCULUS KERATOGRAPH (poster) }\end{array}$ \\
\hline $\begin{array}{l}\text { Chalmers, R. L.; Keay, L.; Kern, J.; } \\
\text { Jansen, M.; Lam, D.; Kinoshita, B.; } \\
\text { Wagner, H.; Sorbara, L.; Bullimore, } \\
\text { M.; Shovlin, J.; Sczcotka-Flynn, L. }\end{array}$ & $\begin{array}{l}\text { CHARACTERIZING CORNEAL INFILTRATES } \\
\text { FROM A } 2010 \text { CASE CONTROL STUDY OF } \\
\text { SOFT CONTACT LENS WEARERS (paper) }\end{array}$ \\
\hline $\begin{array}{l}\text { Sarah Maclver (first author) } \\
\text { Bass S., Sherman J. (SUNY State } \\
\text { College of Optometry) }\end{array}$ & $\begin{array}{l}\text { VASO-OBLITERATION IN A CASE OF IDIO- } \\
\text { PATHIC RETINAL VASCULITIS, ANEURYSMS } \\
\text { AND NEURORETINITIS (poster) }\end{array}$ \\
\hline $\begin{array}{l}\text { Sarah Maclver (first author) } \\
\text { Madonna R., Slotnik S., } \\
\text { Sherman J. (SUNY State College } \\
\text { of Optometry) }\end{array}$ & $\begin{array}{l}\text { SD-OCT IMAGES THE RECOVERY OF } \\
\text { PHOTORECEPTOR FUNCTION IN CASE OF } \\
\text { COMMOTIO RETINAE OF THE MACULA } \\
\text { (poster) }\end{array}$ \\
\hline $\begin{array}{l}\text { Jill Woods (first author) } \\
\text { Ping Situ, Nancy Keir, Craig } \\
\text { Woods, Desmond Fonn }\end{array}$ & $\begin{array}{l}\text { HOW DOES READING ADDITION INFLUENCE } \\
\text { THE PERFORMANCE AND ACCEPTANCE OF } \\
\text { SOFT MULTIFOCAL LENSES? (poster) }\end{array}$ \\
\hline $\begin{array}{l}\text { Jill Woods (first author) } \\
\text { Ping Situ, Craig Woods, } \\
\text { Desmond Fonn }\end{array}$ & $\begin{array}{l}\text { MEDIUM-ADDITION, CENTRE- } \\
\text { NEAR,SILICONE HYDROGEL MULTIFOCAL } \\
\text { LENS COMPARED TO MONOVISION: } \\
\text { PERFORMANCE, ADAPTATION AND } \\
\text { PREFERENCE (poster) }\end{array}$ \\
\hline $\begin{array}{l}\text { Lyndon Jones (first author) } \\
\text { Mark Willcox, Loretta Szczotka- } \\
\text { Flynn, Joe Shovlin, Jason Nichols }\end{array}$ & $\begin{array}{l}\text { BIOFILMS AND BIODEPOSITS: CLINICAL } \\
\text { IMPLICATIONS (paper) }\end{array}$ \\
\hline $\begin{array}{l}\text { Amir Moezzi (first author) } \\
\text { Ping Situ, Doerte Luensmann, } \\
\text { Desmond Fonn, Craig Woods, } \\
\text { John McNally, Lyndon Jones }\end{array}$ & $\begin{array}{l}\text { DOES COMFORT WITH AGING SILICONE } \\
\text { HYDROGEL LENSES RELATE TO CHANGES IN } \\
\text { LENS FIT AND CONJUNCTIVAL STAINING? } \\
\text { (poster) }\end{array}$ \\
\hline $\begin{array}{l}\text { Nancy Keir (first author) } \\
\text { D Richter, CA Woods, } \\
\text { P Bergenske, M Fahmy, } \\
\text { D Luensmann, M Despres, } \\
\text { D Fonn }\end{array}$ & $\begin{array}{l}\text { THE EFFECT OF MASKING ON SUBJECTIVE } \\
\text { RESULTS WITH DAILY DISPOSABLE CONTACT } \\
\text { LENSES (paper) }\end{array}$ \\
\hline $\begin{array}{l}\text { Ping Situ (first author) } \\
\text { Trefford Simpson, Marc Schulze, } \\
\text { Kara Menzies, Hendrik Walther, } \\
\text { Lyndon Jones }\end{array}$ & $\begin{array}{l}\text { INTRA- AND INTER-OPERATOR VARIABILITY } \\
\text { OF MERIDIONAL CORNEAL AND EPITHELIAL } \\
\text { THICKNESS MEASUREMENTS OBTAINED } \\
\text { USING OPTICAL COHERENCE TOMOGRAPHY } \\
\text { (OCT) (poster) }\end{array}$ \\
\hline $\begin{array}{l}\text { Lindsay Paquette (first author) } \\
\text { Debbie Jones, Megan Despres, } \\
\text { Krithika Nandakumar, } \\
\text { Craig Woods }\end{array}$ & $\begin{array}{l}\text { EASE OF CONTACT LENS FITTING AND } \\
\text { TRAINING IN A CHILD AND YOUTH } \\
\text { POPULATION (poster) }\end{array}$ \\
\hline
\end{tabular}

